\title{
On the Fault Tolerant Partition Resolvability of Toeplitz Networks
}

\author{
Asim Nadeem $\mathbb{D},{ }^{1}$ Agha Kashif $\mathbb{D},{ }^{1}$ Amer Aljaedi $\mathbb{D}{ }^{2}$ and Sohail Zafar $\mathbb{D}^{1}$ \\ ${ }^{1}$ Department of Mathematics, University of Management and Technology, Lahore, Pakistan \\ ${ }^{2}$ College of Computing and Information Technology, University of Tabuk, Tabuk 71 491, Saudi Arabia \\ Correspondence should be addressed to Agha Kashif; kashif.khan@umt.edu.pk
}

Received 28 October 2021; Revised 17 December 2021; Accepted 22 December 2021; Published 17 January 2022

Academic Editor: Shaojian Qu

Copyright ( $\odot 2022$ Asim Nadeem et al. This is an open access article distributed under the Creative Commons Attribution License, which permits unrestricted use, distribution, and reproduction in any medium, provided the original work is properly cited.

In any interconnection network, fault tolerance is the most desirable property to achieve reliability. Toeplitz networks are used as interconnection networks due their smaller diameter, symmetry, simpler routing, high connectivity, and reliability. The partition dimension of a network is presented as an extension of metric dimension of networks. Its applications can be seen in several areas including robot navigation, network designing, image processing, and chemistry. In this article, the fault tolerant partition dimension, $\operatorname{~pd}_{2}\left(T_{n}\langle 1, t\rangle\right)$, of Toeplitz networks, is shown to be bounded below by 4 for $t \geq 2, n \geq 4$, whereas it is bounded above by 5 for $t=3, n \geq 14$. Further, it is shown that the exact value of $\operatorname{pd}_{2}\left(T_{n}\langle 1, t\rangle\right)$ equals 4 for $t=2, n \geq 4 ; t=3, n \in\{5,6, \ldots, 13\}$; and $t \geq 4, n \in\{t+2, t+3, t+4\}$.

\section{Introduction}

Computer networking gives a way of communication among several processors of a computer and numerous computers connected to a network. The objective of the interconnection networks is to reduce the time when it becomes impossible to manage large amount of data by a single processor, so the job is divided among the several processors working at the same time. Effective interconnection networks play vital rule in transferring data briskly among the different components of parallel processing. It is always preferable for a computer network to have smaller diameter, lesser degree, alternate paths among the nodes, higher level of symmetry, simpler routing, and high level of connectivity. Fault tolerance of a network ensures that the system will continue to work even if some of its components fail to work. This also guarantees the lower maintenance cost and longer durability of the network. Toeplitz networks are used as interconnection networks due their smaller diameter, symmetry, simpler routing, high connectivity, and reliability [1]. In this article, we have computed the fault tolerant partition dimension of some particular classes of Toeplitz networks.
1.1. Background and Related Work. The metric basis is a set that contains least number of nodes of a network such that all nodes are exclusively identified by the distances from nodes in the basis. Motivated by the need of such a set of nodes, Slater [2] and Harary and Melter [3] independently introduced the concept of metric basis in 1975 and 1976. Slater [2] used metric basis to locate the invader in the given network. Khuller et al. [4] used it for robot navigation in 1996. In 2000, Chartrand et al. [5] used it to locate position of functional groups in chemical compounds. In 2008, Hernando et al. [6] presented the notion of fault tolerant metric dimension of graphs. In 2015, Estrado-Moreno et al. [7] presented the concept of $\kappa$-metric. For $\kappa=2$, the $\kappa$-metric dimension is known as fault tolerant metric dimension denoted by $\operatorname{dim}_{2}(W)$. Further study on $\kappa$-metric dimension can be seen in $[8,9]$.

An important variant of metric basis is the partition basis which is the partition of the nodes of a network with minimum size such that all nodes are exclusively identified by the distances from sets in the partition. The minimum size of a resolving partition is called partition dimension of the network. In 2000, Chartrand et al. [10] presented the concept of partition dimension which is an extension of metric 
dimension. In 2009, Javaid et al. [11] introduced the concept of fault tolerant partition dimension. In 2014, the partition dimension of unicyclic graphs was discussed in [12]. The partition dimension of some families of trees was studied by Fredlina and Baskaro [13] in 2015. In 2020, Estrado-Moreno [14] presented the concept of $\kappa$-partition dimension of the network. For $\kappa=2$, the $\kappa$-partition dimension is known as fault tolerant partition dimension denoted by $\operatorname{pd}_{2}(W)$. The $\operatorname{pd}_{2}(W)$ was computed for some important networks in $[15,16]$.

Harary and Melter in [3] concluded that computation of metric basis of a network is an NP-hard problem; it further implies that computation of partition-related metric parameters for general graphs is also NP-hard. This lead the current research trends to focus on the computation of these metric parameters for specific classes of graphs. Recently, Liu et al. in [17] calculated the metric dimension of Toeplitz networks. The present research on Toeplitz networks and their merits in the interconnection networks [1] motivated us to study fault tolerant partition dimension of Toeplitz networks.

1.2. Preliminaries. Considering a connected network $W$, the node and edge sets of $W$ are denoted by $V(W)$ and $E(W)$, respectively. The length of the shortest path, connecting two nodes and $q$, is known as the distance between $p$ and $q$ denoted by $d(p, q)$. The distance of a node $p$ from a subset $A$ of the node set is $d(p, A)=\min \{d(p, t) \mid t \in A\}$, whereas the set $N(p)=\{q \in V(G) \mid p q \in E(G)\}$ is called the neighbourhood of node $p$. Let $\Omega=\left\{x_{1}, x_{2}, \ldots, x_{l}\right\}$ be an ordered set of nodes, the representation of a node $p$ with respect to $\Omega$ is a vector $r(p \mid \Omega)$ such that $r(p \mid \Omega)=\left(d\left(p, x_{1}\right)\right.$, $\left.d\left(p, x_{2}\right), \ldots, d\left(p, x_{l}\right)\right)$. If the vectors $r(p \mid \Omega)$ are different, for every node of the network, then $\Omega$ is termed as a resolving set of $W$. The minimal cardinality of $\Omega$ is termed as the metric dimension of $W$, represented by $\operatorname{dim}(W)$. If the vectors $r(p \mid \Omega)$ are different in at least $\kappa$ places for every node of the graph, then $\Omega$ is termed as a $\kappa$-metric generator for $W$. $\kappa-$ metric basis is a generator with least number of nodes. The minimal cardinality of $\Omega$ is termed as the $\kappa$-metric dimension of $W$, represented by $\operatorname{dim}_{\kappa}(W)$.

Let $\Psi=\left\{B_{1}, B_{2}, \ldots, B_{l}\right\}$ be an ordered $l$-partition of a connected network $W$. The partition representation of node $p$ with respect to $\Psi$ is a vector $r(p \mid \Psi)$ such that $r(p \mid \Psi)=\left(d\left(p, B_{1}\right), d\left(p, B_{2}\right), \ldots, d\left(p, B_{l}\right)\right)$. If the vectors $r(p \mid \Psi)$ are different for every node of the graph, then $l$-partition is termed as a resolving partition of $W$. The minimal cardinality of $\Psi$ for which there is a resolving $l$-partition is termed as the partition dimension of $W$, represented by $\mathrm{pd}(W)$. If the vectors $r(p \mid \Psi)$ are different in at least $\kappa$ places for every node of the network, then $\Psi$ is termed as a $\kappa$-partition generator of $W$. $\mathcal{\kappa}$-partition basis is a generator with least number of sets. The minimal cardinality of $\Psi$ is termed as a the $\kappa$-partition dimension of $W$, represented by $\operatorname{pd}_{\kappa}(W)$.

1.3. Main Results. The study conducted in this paper leads to the following results.
Theorem 1. Let $T_{n}\langle 1, t\rangle$ be the Toeplitz network, then

(1) For $n \geq 4, p d_{2}\left(T_{n}\langle 1, t\rangle\right) \geq 4$

(2) For $n \geq 4$, $p d_{2}\left(T_{n}\langle 1,2\rangle\right)=4$

(3) For $n \in\{5,6, \ldots, 13\}$, $p d_{2}\left(T_{n}\langle 1,3\rangle\right)=4$

(4) For $n \geq 14,4 \leq p d_{2}\left(T_{n}\langle 1,3\rangle\right) \leq 5$

(5) For $t \geq 5$ and $n=t+2, t+3, t+4, p d_{2}\left(T_{n}\langle 1, t\rangle\right)=4$

In Section 2, Toeplitz networks are defined and the fault tolerant partition dimension of Toeplitz networks, $T_{n}\langle 1, t\rangle$, for different values of $n$ and $t$ have been computed. In Section 3 , the results are summarized and two open problems are proposed.

\section{Fault Tolerant Partition Dimension of Toeplitz Networks}

For a sequence of positive integers, $n, k_{1}, k_{2}, \ldots, k_{n}$ with $k_{1}, k_{2}, \ldots, k_{n} \leq n-1$, the Toeplitz network $T_{n}\left\langle k_{1}\right.$, $\left.k_{2}, \ldots, k_{n}\right\rangle$ is a network on the vertex set $\left\{x_{1}, x_{2}, \ldots, x_{n}\right\}$ with two vertices $x_{i}$ and $x_{j}$ which are adjacent if and only if $|i-j| \in\left\{k_{1}, k_{2}, \ldots, k_{n}\right\}$. Figure 1 shows $T_{14}\langle 1,3\rangle$.

The following lemma gives the lower bound on the fault tolerant partition dimension of Toeplitz networks $T_{n}\langle 1, t\rangle$.

Lemma 1. Let $T_{n}\langle 1, t\rangle$ be the Toeplitz network with $n \geq 4$, then $p d_{2}\left(T_{n}\langle 1, t\rangle\right) \geq 4$.

Proof. Assume that $\operatorname{pd}_{2}\left(T_{n}\langle 1, t\rangle\right)=3$ for $n \geq 4$. Let $\Psi=\left\{A_{1}, A_{2}, A_{3}\right\}$ be a fault tolerant partition basis of $V\left(T_{n}\langle 1, t\rangle\right)$. Clearly, one of the sets $A_{1}, A_{2}$, and $A_{3}$ contains at least two vertices. Suppose that $\left|A_{1}\right| \geq 2$. Here, at least one node of $A_{1}$, say $v_{k}$, has a coordinate of $r\left(v_{k} \mid \Psi\right)>1$. Otherwise, each node in $A_{1}$ will have the same representation. So, without loss of generality, we can assume that the third coordinate of $r\left(v_{k} \mid \Psi\right)$ is greater than 1. i.e., $d\left(v_{k}, A_{3}\right) \geq 2$. Let $v_{m}$ be a node in $A_{3}$ such that $d\left(v_{k}, v_{m}\right)=d\left(v_{k}, A_{3}\right)$. Let $N\left(v_{k}\right)$ be the neighbourhood of $v_{k}$. So, $N\left(v_{k}\right) \cap A_{3}=\varnothing$ as $d\left(u, v_{k}\right)<d\left(v_{k}, v_{m}\right)$ for all $u \in N\left(v_{k}\right)$. Since $2 \leq\left|N\left(v_{k}\right)\right| \leq 4$ in the $T_{n}\langle 1, t\rangle$ Toeplitz network, so we have the following three cases.

Case 1: when $\left|N\left(v_{k}\right)\right|=4$, let $N\left(v_{k}\right)=\left\{x_{1}, x_{2}, x_{3}, x_{4}\right\}$.

Case 1.1: suppose all the nodes of $N\left(v_{k}\right)$ are in the same set $A_{1}$ or $A_{2}$. Assume that $N\left(v_{k}\right) \subseteq A_{1}$, then $r\left(v_{k} \mid \Psi\right)=\left(0, a_{0}, b_{0}\right)$ and for $x \in N\left(v_{k}\right), r\left(x_{l} \mid \Psi\right)=$ $\left(0, a_{l}, b_{l}\right)$, for $1 \leq l \leq 4$. Hence, $a_{0}-1 \leq a_{1}, a_{2}$, $a_{3}, a_{4} \leq a_{0}+1$. Now, pigeonhole principle implies that at least two of the nodes $v_{k}$ and $x_{l}$ will be equidistant from $A_{1}$ and $A_{2}$, which is a contradiction, whereas $N\left(v_{k}\right) \subseteq A_{2}$ generates a trivial case of contradiction.

Case 1.2: assume that $A_{1}$ or $A_{2}$ contains three vertices from $N\left(v_{k}\right)$.

Let $x_{p}, x_{q}, x_{r} \in N\left(v_{k}\right) \cap A_{1}$, and $x_{t} \in N\left(v_{k}\right) \cap A_{2}$, then $r\left(v_{k} \mid \Psi\right)=\left(0,1, b_{0}\right), \quad r\left(x_{p} \mid \Psi\right)=\left(0, a_{1}, b_{1}\right)$, $r\left(x_{q} \mid \Psi\right)=\left(0, a_{2}, b_{2}\right)$, and $r\left(x_{q} \mid \Psi\right)=\left(0, a_{3}, b_{3}\right)$. In this case, we have $1 \leq a_{1}, a_{2}, a_{3} \leq 2$. Again, pigeonhole principle implies that at least two of the nodes $v_{k}, x_{p}, x_{q}$, and $x_{r}$ will be equidistant from $A_{1}$ and $A_{2}$, 


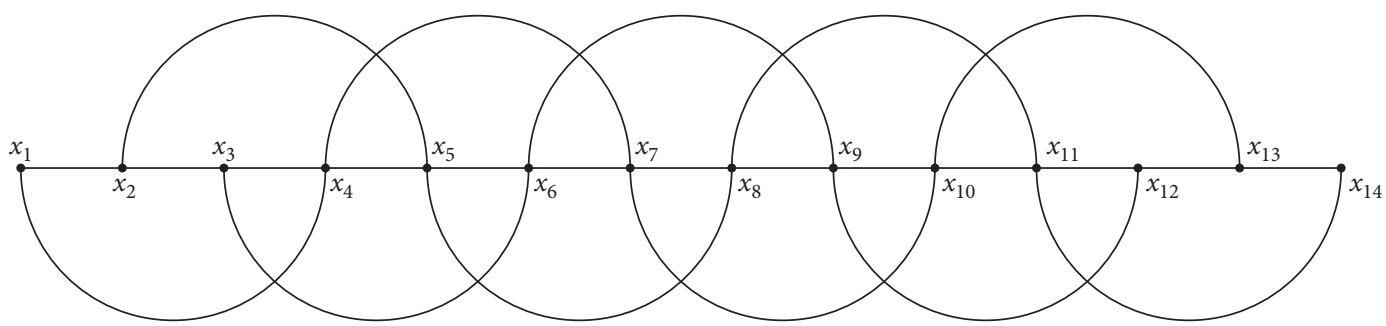

Figure 1: Toeplitz network $T_{14}\langle 1,3\rangle$.

leading to a contradiction, whereas three nodes of $N\left(v_{k}\right)$ in $A_{2}$ generate a trivial case of contradiction. Case 1.3: assume that each of $A_{1}$ and $A_{2}$ contains two nodes of $N\left(v_{k}\right)$. Let $x_{p}, x_{q} \in N\left(v_{k}\right) \cap A_{1}$ and $x_{r}, x_{t} \in N\left(v_{k}\right) \cap A_{2}$, then $r\left(v_{k} \mid \Psi\right)=\left(0,1, b_{0}\right)$, $r\left(x_{p} \mid \Psi\right)=\left(0, a_{1}, b_{1}\right)$ and $r\left(x_{q} \mid \Psi\right)=\left(0, a_{2}, b_{2}\right)$. In this case, we have $1 \leq a_{1}, a_{2} \leq 2$. Again by pigeonhole principle, at least two of the nodes $v_{k}, x_{p}$ and $x_{q}$ will have the same distances from $A_{1}$ and $A_{2}$. This leads to a contradiction. Now, if we consider nodes in $A_{2}$, then $r\left(x_{r} \mid \Psi\right)=\left(1,0, b_{1}\right)$ and $r\left(x_{t} \mid \Psi\right)=\left(1,0, b_{2}\right)$, which is again a contradiction.

Case 2: when $\left|N\left(v_{k}\right)\right|=3$, let $N\left(v_{k}\right)=\left\{x_{1}, x_{2}, x_{3}\right\}$. The subcases in this case are similar to Case 1.2 and Case 1.3 .

Case 3: when $\left|N\left(v_{k}\right)\right|=2$, let $N\left(v_{k}\right)=\left\{x_{1}, x_{2}\right\}$.

Case 3.1: if both of the nodes of $N\left(v_{k}\right)$ are in the same set $A_{1}$ or $A_{2}$, assume that $N\left(v_{k}\right) \subseteq A_{1}$, then $r\left(v_{k} \mid \Psi\right)=$ $\left(0, a_{0}, b_{0}\right), r\left(x_{p} \mid \Psi\right)=\left(0, a_{1}, b_{1}\right) \quad$ and $r\left(x_{q} \mid \Psi\right)=\left(0, a_{2}, b_{2}\right)$. Hence, $a_{0}-1 \leq a_{1}, a_{2} \leq a_{0}+1$. Now, if any of $a_{1}$ and $a_{2}$ is equal to $a_{0}$, then we have a contradiction. Since in Toeplitz networks the only nodes with two neighbours are $v_{1}$ and $v_{n}$, so in all other cases, we will have $3 \leq\left|N\left(v_{p}\right)\right| \leq 4$ and $3 \leq\left|N\left(v_{q}\right)\right| \leq 4$ which are similar to Case 1 and Case 2, whereas $N\left(v_{k}\right) \subseteq A_{2}$ generates a trivial case of contradiction.

Case 3.2: assume that each of $A_{1}$ and $A_{2}$ contains a node from the set $N\left(v_{k}\right)$. Let $x_{p} \in N\left(v_{k}\right) \cap A_{1}$ and $x_{q} \in N\left(v_{k}\right) \cap A_{2}$, then we can consider the $N\left(v_{p}\right)$ and $N\left(v_{q}\right)$ to get contradiction as in the previous cases.

Hence, $\operatorname{pd}_{2}\left(T_{n}\langle 1, t\rangle\right) \geq 4$ for $n \geq 4$.

In the following theorems, we calculate the fault tolerant partition dimension of Toeplitz networks $T_{n}\langle 1, t\rangle$ for different values of $t$.

Theorem 2. Let $T_{n}\langle 1,2\rangle$ be the Toeplitz network with $n \geq 4$, then $p d_{2}\left(T_{n}\langle 1,2\rangle\right)=4$.

Proof. Let $\Psi=\left\{A_{1}, A_{2}, A_{3}, A_{4}\right\}$ be a partition of $V\left(T_{n}\langle 1,2\rangle\right)$. The proof is divided into three cases, and in each case, we will show that $\Psi$ is the fault tolerant partition generator of $T_{n}\langle 1,2\rangle$. Let $\kappa=\lceil n / 2\rceil$.
Case 1: for $\kappa=3\rangle$, let $A_{1}=\left\{v_{2}, v_{4}, \ldots, v_{2(\lfloor n / 2\rfloor)}\right\}$, $A_{2}=\left\{v_{1}, v_{3}, \ldots, v_{2\rangle-1}\right\}, \quad A_{3}=\left\{v_{2\rangle+1}, v_{2\rangle+3}, \ldots, v_{4\rangle-1}\right\}$, and $A_{4}=\left\{v_{4\rangle+1}, v_{4\rangle+3}, \ldots, v_{2 \kappa-1}\right\}$.

When $\kappa=3\rangle$, the $r\left(v_{a} \mid \Psi\right)$ are tabulated in Table 1 .

Case 2: for $\kappa=3\rangle+1$, let $A_{1}=\left\{v_{2}, v_{4}, \ldots, v_{2(\lfloor n / 2\rfloor)}\right\}$, $A_{2}=\left\{v_{1}, v_{3}, \ldots, v_{2\lfloor\kappa / 3\rfloor+1}\right\}, A_{3}=\left\{v_{2\lfloor\kappa / 3\rfloor+3}, v_{2\lfloor\kappa / 3\rfloor+5}, \ldots\right.$, $\left.v_{4\lfloor\kappa / 3\rfloor+1}\right\}$, and $A_{4}=\left\{v_{4\lfloor\kappa / 3\rfloor+3}, v_{4\lfloor\kappa / 3\rfloor+5}, \ldots, v_{2 \kappa-1}\right\}$

When $\kappa=3\rangle+1$, the $r\left(v_{a} \mid \Psi\right)$ are tabulated in Table 2 .

Case 3: for $\kappa=3 \varrho+2$, let $A_{1}=\left\{v_{2}, v_{4}, \ldots, v_{2(\lfloor n / 2\rfloor)}\right\}$, $A_{2}=\left\{v_{1}, v_{3}, \ldots, v_{2\lceil\kappa / 3\rceil-1}\right\}, \quad A_{3}=\left\{v_{2\lceil\kappa / 3\rceil+1}, v_{2\lceil\kappa / 3\rceil+3}\right.$, $\left.\ldots, v_{4\lceil\kappa / 3\rceil-1}\right\}$, and $A_{4}=\left\{v_{4\lceil\kappa / 3\rceil+1}, v_{4\lceil\kappa / 3\rceil+3}, \ldots, v_{2 \kappa-1}\right\}$. When $\kappa=3\rangle+2$, the $r\left(v_{a} \mid \Psi\right)$ are tabulated in Table 3 .

Tables $1-3$ clearly show that $\Psi$ is a $2-$ resolving generator of $T_{n}\langle 1,2\rangle$ for $n \geq 4$, therefore $\operatorname{pd}_{2}\left(T_{n}\langle 1,2\rangle\right) \leq 4$ for $n \geq 4$. Lemma 1 implies that $\operatorname{pd}_{2}\left(T_{n}\langle 1,2\rangle\right) \geq 4$ for $n \geq 4$. This establishes our claim.

Theorem 3. Let $T_{n}\langle 1,3\rangle$ be the Toeplitz network, then $p d_{2}\left(T_{n}\langle 1,3\rangle\right)=4 \quad$ when $n \in\{5,6, \ldots, 13\}$ and $4 \leq p d_{2}\left(T_{n}\langle 1,3\rangle\right) \leq 5$ for $n \geq 14$.

Proof. The proof is divided into two parts.

Case 1: the partition $\Psi$ for $5 \leq n \leq 13$ is tabulated in Table 4 which clearly shows that $\Psi$ is the fault tolerant partition generator; therefore, $\operatorname{pd}_{2}\left(T_{n}\langle 1,3\rangle\right) \leq 4$ for $5 \leq n \leq 13$. Lemma limplies that $\operatorname{pd}_{2}\left(T_{n}\langle 1,3\rangle\right) \geq 4$ for $5 \leq n \leq 13$. This establishes our claim.

Case 2: suppose $\Psi=\left\{A_{1}, A_{2}, A_{3}, A_{4}, A_{5}\right\}$ be a partition of $V\left(T_{n}\langle 1,3\rangle\right)$ for $n \geq 14$. Let $A_{1}=\left\{v_{1}, v_{2}, \ldots, v_{n-6}\right\}$, $A_{2}=\left\{v_{n-5}, v_{n-4}\right\}, A_{3}=\left\{v_{n-3}, v_{n-2}\right\}, A_{4}=\left\{v_{n-1}\right\}, \quad$ and $A_{5}=\left\{v_{n}\right\}$. For $\left.\left.\left.n=3\right\rangle, 3\right\rangle+1,3\right\rangle+2$, the $r\left(v_{a} \mid \Psi\right)$ are tabulated in Tables 5-7, respectively.

Tables 5-7 clearly show that $\Psi$ is a $2-$ resolving generator of $T_{n}\langle 1,3\rangle$ for $n \geq 14$, so $\operatorname{pd}_{2}\left(T_{n}\langle 1,3\rangle\right) \leq 5$ for $n \geq 14$. Lemma 1 implies that $\operatorname{pd}_{2}\left(T_{n}\langle 1,3\rangle\right) \geq 4$ for $n \geq 14$. This establishes that $4 \leq \operatorname{pd}_{2}\left(T_{n}\langle 1,3\rangle\right) \leq 5$.

Theorem 4. Let $T_{n}\langle 1, t\rangle$ be the Toeplitz network with odd $t \geq 5$ and $n=t+2, t+3, t+4$, then $p d_{2}\left(T_{n}\langle 1, t\rangle\right)=4$.

Proof. Let $\Psi=\left\{A_{1}, A_{2}, A_{3}, A_{4}\right\} \quad$ be a partition of $V\left(T_{n}\langle 1, t\rangle\right)$. The proof consists of three parts. 
TABLe 1: $r\left(v_{a} \mid \Psi\right)$ for $T_{n}\langle 1,2\rangle$ when $\left.\kappa=3\right\rangle$.

\begin{tabular}{|c|c|c|c|c|}
\hline Nodes & $d\left(v_{a}, A_{1}\right)$ & $d\left(v_{a}, A_{2}\right)$ & $d\left(v_{a}, A_{3}\right)$ & $d\left(v_{a}, A_{4}\right)$ \\
\hline$\left.v_{2 a}(1 \leq a \leq\rangle\right)$ & 0 & 1 & $>-a+1$ & 2) $-a+1$ \\
\hline$\left.v_{2 a}(>+1 \leq a \leq 2\rangle\right)$ & 0 & $a->+1$ & 1 & 2) $-a+1$ \\
\hline$\left.v_{2 a}(2\rangle+1 \leq a \leq\lfloor n / 2\rfloor\right)$ & 0 & $a->+1$ & $a-2\rangle+1$ & 1 \\
\hline$v_{2 a+1}(0 \leq a \leq>-1)$ & 1 & 0 & $>-a$ & $2>-a$ \\
\hline$\left.\left.v_{2 a+1}(\rangle \leq a \leq 2\right\rangle-1\right)$ & 1 & $a->+1$ & 0 & $2\rangle-a$ \\
\hline$\left.v_{2 a+1}(2\rangle \leq a \leq \kappa-1\right)$ & 1 & $a->+1$ & $a-2\rangle+1$ & 0 \\
\hline
\end{tabular}

TABLe 2: $r\left(v_{a} \mid \Psi\right)$ for $T_{n}\langle 1,2\rangle$ when $\left.\kappa=3\right\rangle+1$.

\begin{tabular}{|c|c|c|c|c|}
\hline Nodes & $d\left(v_{a}, A_{1}\right)$ & $d\left(v_{a}, A_{2}\right)$ & $d\left(v_{a}, A_{3}\right)$ & $d\left(v_{a}, A_{4}\right)$ \\
\hline$v_{2 a}(1 \leq a \leq>+1)$ & 0 & 1 & $>-a+2$ & $2>-a+2$ \\
\hline$\left.v_{2 a}(>+2 \leq a \leq 2\rangle+1\right)$ & 0 & $a->$ & 1 & $2\rangle-a+2$ \\
\hline$\left.v_{2 a}(2\rangle+2 \leq a \leq\lfloor n / 2\rfloor\right)$ & 0 & $a->$ & $a-2\rangle$ & 1 \\
\hline$\left.v_{2 a+1}(0 \leq a \leq\rangle\right)$ & 1 & 0 & $>-a+1$ & $2\rangle-a+1$ \\
\hline$\left.\left.v_{2 a+1}(\rangle+1 \leq a \leq 2\right\rangle\right)$ & 1 & $a->$ & 0 & $2>-a+1$ \\
\hline$\left.v_{2 a+1}(2\rangle+1 \leq a \leq \kappa-1\right)$ & 1 & $a->$ & $a-2\rangle$ & 0 \\
\hline
\end{tabular}

TABLe 3: $r\left(v_{a} \mid \Psi\right)$ for $T_{n}\langle 1,2\rangle$ when $\left.\kappa=3\right\rangle+2$.

\begin{tabular}{|c|c|c|c|c|}
\hline Nodes & $d\left(v_{a}, A_{1}\right)$ & $d\left(v_{a}, A_{2}\right)$ & $d\left(v_{a}, A_{3}\right)$ & $d\left(v_{a}, A_{4}\right)$ \\
\hline$v_{2 a}(1 \leq a \leq>+1)$ & 0 & 1 & $>-a+2$ & $2>-a+3$ \\
\hline$\left.v_{2 a}(>+2 \leq a \leq 2\rangle+2\right)$ & 0 & $a->$ & 1 & 2) $-a+3$ \\
\hline$\left.v_{2 a}(2\rangle+3 \leq a \leq\lfloor n / 2\rfloor\right)$ & 0 & $a->$ & $a-2\rangle-1$ & 1 \\
\hline$\left.v_{2 a+1}(0 \leq a \leq\rangle\right)$ & 1 & 0 & $>-a+1$ & $2\rangle-a+2$ \\
\hline$\left.\left.v_{2 a+1}(\rangle+1 \leq a \leq 2\right\rangle+1\right)$ & 1 & $a->$ & 0 & 2) $-a+2$ \\
\hline$\left.v_{2 a+1}(2\rangle+2 \leq a \leq \kappa-1\right)$ & 1 & $a->$ & $a-2>-1$ & 0 \\
\hline
\end{tabular}

TABle 4: Partitions for $n=5,6, \ldots, 13$.

\begin{tabular}{lcccc}
\hline$n$ & $A_{1}$ & $A_{2}$ & $A_{3}$ & $A_{4}$ \\
\hline 5 & $v_{1}, v_{2}$ & $v_{3}$ & $v_{4}$ & $v_{5}$ \\
6 & $v_{1}, v_{2}$ & $v_{3}, v_{4}$ & $v_{6}$ & $v_{5}$ \\
7 & $v_{1}, v_{2}$ & $v_{3}$ & $v_{6}$ & $v_{8}$ \\
8 & $v_{1}, v_{2}, v_{5}$ & $v_{3}, v_{4}, v_{7}$ & $v_{5}, v_{8}, v_{9}$ & $v_{9}, v_{10}$ \\
9 & $v_{1}, v_{2}, v_{5}$ & $v_{3}, v_{4}, v_{7}$ & $v_{5}, v_{6}, v_{7}$ & $v_{9}, v_{10}, v_{11}$ \\
10 & $v_{1}, v_{2}, v_{3}$ & $v_{4}, v_{8}$ & $v_{5}, v_{6}, v_{7}$ & $v_{9}, v_{10}, v_{11}$ \\
11 & $v_{1}, v_{2}, v_{3}$ & $v_{4}, v_{8}$ & $v_{5}, v_{6}, v_{7}$ & $v_{8}, v_{9}, v_{10}$ \\
12 & $v_{1}, v_{2}, v_{3}$ & $v_{4}, v_{8}, v_{12}$ & $v_{4}, v_{5}, v_{6}$ & \\
13 & $v_{1}, v_{2}, v_{12}, v_{13}$ & $v_{3}, v_{7}, v_{11}$ & & \\
\hline
\end{tabular}

TABLE 5: $r\left(v_{a} \mid \Psi\right)$ for $T_{n}\langle 1,3\rangle$ when $\left.n=3\right\rangle$.

\begin{tabular}{|c|c|c|c|c|c|}
\hline Nodes & $d\left(v_{a}, A_{1}\right)$ & $d\left(v_{a}, A_{2}\right)$ & $d\left(v_{a}, A_{3}\right)$ & $d\left(v_{a}, A_{4}\right)$ & $d\left(v_{a}, A_{5}\right)$ \\
\hline$v_{3 a+1}(0 \leq a \leq>-3)$ & 0 & $>-a-2$ & $>-a-1$ & $>-a$ & $>-a+1$ \\
\hline$v_{3 a-1}(1 \leq a \leq>-2)$ & 0 & $>-a-1$ & $>-a$ & $>-a$ & $>-a+1$ \\
\hline$v_{3 a}(1 \leq a \leq>-2)$ & 0 & $>-a-1$ & $>-a-1$ & $>-a+1$ & $>-a$ \\
\hline$v_{a}(a=n-5)$ & 1 & 0 & 1 & 2 & 3 \\
\hline$v_{a}(a=n-4)$ & 1 & 0 & 1 & 1 & 2 \\
\hline$v_{a}(a=n-3)$ & 1 & 1 & 0 & 2 & 1 \\
\hline$v_{a}(a=n-2)$ & 2 & 1 & 0 & 1 & 2 \\
\hline$v_{a}(a=n-1)$ & 2 & 1 & 1 & 0 & 1 \\
\hline$v_{a}(a=n)$ & 2 & 2 & 1 & 1 & 0 \\
\hline
\end{tabular}


TABLe 6: $r\left(v_{a} \mid \Psi\right)$ for $T_{n}\langle 1,3\rangle$ when $\left.n=3\right\rangle+1$.

\begin{tabular}{|c|c|c|c|c|c|}
\hline Nodes & $d\left(v_{a}, A_{1}\right)$ & $d\left(v_{a}, A_{2}\right)$ & $d\left(v_{a}, A_{3}\right)$ & $d\left(v_{a}, A_{4}\right)$ & $d\left(v_{a}, A_{5}\right)$ \\
\hline$v_{3 a+1}(0 \leq a \leq>-2)$ & 0 & $>-a-1$ & $>-a$ & $>-a$ & $>-a+1$ \\
\hline$v_{3 a-1}(1 \leq a \leq>-2)$ & 0 & $>-a-1$ & $>-a$ & $>-a+1$ & $>-a+2$ \\
\hline$v_{3 a}(1 \leq a \leq>-2)$ & 0 & $>-a-1$ & $>-a$ & $>-a$ & $>-a+1$ \\
\hline$v_{a}(a=n-5)$ & 1 & 0 & 1 & 2 & 3 \\
\hline$v_{a}(a=n-4)$ & 1 & 0 & 1 & 1 & 2 \\
\hline$v_{a}(a=n-3)$ & 1 & 1 & 0 & 2 & 1 \\
\hline$v_{a}(a=n-2)$ & 2 & 1 & 0 & 1 & 2 \\
\hline$v_{a}(a=n-1)$ & 2 & 1 & 1 & 0 & 1 \\
\hline$v_{a}(a=n)$ & 2 & 2 & 1 & 1 & 0 \\
\hline
\end{tabular}

TABLE 7: $r\left(v_{a} \mid \Psi\right)$ for $T_{n}\langle 1,3\rangle$ when $\left.n=3\right\rangle+2$.

\begin{tabular}{|c|c|c|c|c|c|}
\hline Nodes & $d\left(v_{a}, A_{1}\right)$ & $d\left(v_{a}, A_{2}\right)$ & $d\left(v_{a}, A_{3}\right)$ & $d\left(v_{a}, A_{4}\right)$ & $d\left(v_{a}, A_{5}\right)$ \\
\hline$v_{3 a+1}(0 \leq a \leq>-2)$ & 0 & $>-a-1$ & $>-a$ & $>-a$ & \rangle$-a+1$ \\
\hline$v_{3 a-1}(1 \leq a \leq>-1)$ & 0 & $>-a$ & $>-a$ & $>-a+2$ & $>-a+1$ \\
\hline$v_{3 a}(1 \leq a \leq>-2)$ & 0 & $>-a-1$ & $>-a$ & $>-a+1$ & $>-a+2$ \\
\hline$v_{a}(a=n-5)$ & 1 & 0 & 1 & 2 & 3 \\
\hline$v_{a}(a=n-4)$ & 1 & 0 & 1 & 1 & 2 \\
\hline$v_{a}(a=n-3)$ & 1 & 1 & 0 & 2 & 1 \\
\hline$v_{a}(a=n-2)$ & 2 & 1 & 0 & 1 & 2 \\
\hline$v_{a}(a=n-1)$ & 2 & 1 & 1 & 0 & 1 \\
\hline$v_{a}(a=n)$ & 2 & 2 & 1 & 1 & 0 \\
\hline
\end{tabular}

TABLE 8: $r\left(v_{a} \mid \Psi\right)$ for $T_{n}\langle 1, t\rangle$ when $n=t+2$ with odd $t \geq 5$.

\begin{tabular}{|c|c|c|c|c|}
\hline Nodes & $d\left(v_{a}, A_{1}\right)$ & $d\left(v_{a}, A_{2}\right)$ & $d\left(v_{a}, A_{3}\right)$ & $d\left(v_{a}, A_{4}\right)$ \\
\hline$v_{a}(a=1)$ & 0 & 2 & 1 & 2 \\
\hline$v_{a}(a=2)$ & 0 & 2 & 2 & 1 \\
\hline$v_{a}(a=3)$ & 0 & 1 & 3 & 2 \\
\hline$v_{a}(4 \leq a \leq n+1 / 2-1)$ & $a-3$ & 0 & $g$ & $a-1$ \\
\hline$v_{a}(a=n+1 / 2)$ & $n+1 / 2-3$ & 0 & $n+1 / 2-2$ & $n+1 / 2-1$ \\
\hline$v_{a}(n+1 / 2+1 \leq a \leq n-2)$ & $n-a$ & 0 & $n-a-1$ & $n-a$ \\
\hline$v_{a}(a=n-1)$ & 1 & 1 & 0 & 1 \\
\hline$v_{a}(a=n)$ & 1 & 2 & 1 & 0 \\
\hline
\end{tabular}

TABLE 9: $r\left(v_{a} \mid \Psi\right)$ for $T_{n}\langle 1, t\rangle$ when $n=t+3$ with odd $t \geq 5$.

\begin{tabular}{|c|c|c|c|c|}
\hline Nodes & $d\left(v_{a}, A_{1}\right)$ & $d\left(v_{a}, A_{2}\right)$ & $d\left(v_{a}, A_{3}\right)$ & $d\left(v_{a}, A_{4}\right)$ \\
\hline$v_{a}(a=1)$ & 0 & 1 & 2 & 3 \\
\hline$v_{a}(a=2)$ & 0 & 2 & 1 & 2 \\
\hline$v_{a}(a=3)$ & 0 & 2 & 2 & 1 \\
\hline$v_{a}(a=4)$ & 0 & 1 & 3 & 2 \\
\hline$v_{a}(5 \leq a \leq n / 2)$ & $a-4$ & 0 & $a-1$ & $a-2$ \\
\hline$v_{a}(a=n / 2+1)$ & $n / 2-3$ & 0 & $n / 2-2$ & $n / 2-1$ \\
\hline$v_{a}(n / 2+2 \leq a \leq n-2)$ & $n-a-1$ & 0 & $n-a-1$ & $n-a$ \\
\hline$v_{a}(a=n-1)$ & 1 & 1 & 0 & 1 \\
\hline$v_{a}(a=n)$ & 1 & 2 & 1 & 0 \\
\hline
\end{tabular}

TABLe 10: $r\left(v_{a} \mid \Psi\right)$ for $T_{n}\langle 1, t\rangle$ when $n=t+4$ with odd $t \geq 5$.

\begin{tabular}{|c|c|c|c|c|}
\hline Nodes & $d\left(v_{a}, A_{1}\right)$ & $d\left(v_{a}, A_{2}\right)$ & $d\left(v_{a}, A_{3}\right)$ & $d\left(v_{a}, A_{4}\right)$ \\
\hline$v_{a}(a=1)$ & 0 & 1 & 2 & 4 \\
\hline$v_{a}(a=2)$ & 0 & 2 & 1 & 3 \\
\hline$v_{a}(a=3)$ & 0 & 1 & 1 & 2 \\
\hline
\end{tabular}


Table 10: Continued.

\begin{tabular}{|c|c|c|c|c|}
\hline Nodes & $d\left(v_{a}, A_{1}\right)$ & $d\left(v_{a}, A_{2}\right)$ & $d\left(v_{a}, A_{3}\right)$ & $d\left(v_{a}, A_{4}\right)$ \\
\hline$v_{a}(4 \leq a \leq n+1 / 2-1)$ & $a-3$ & 0 & $a-2$ & $a-3$ \\
\hline$v_{a}(a=n+1 / 2)$ & $n+1 / 2-3$ & 0 & $n+1 / 2-3$ & $n+1 / 2-3$ \\
\hline$v_{a}(n+1 / 2+1 \leq a \leq n-3)$ & $n-a-2$ & 0 & $n-a-2$ & $n-a$ \\
\hline$v_{a}(a=n-2)$ & 1 & 1 & 0 & 2 \\
\hline$v_{a}(a=n-1)$ & 1 & 2 & 0 & 1 \\
\hline$v_{a}(a=n)$ & 2 & 1 & 1 & 0 \\
\hline
\end{tabular}

TABLe 11: $r\left(v_{a} \mid \Psi\right)$ for $T_{n}\langle 1, t\rangle$ when $n=t+2$ with even $t \geq 4$.

\begin{tabular}{|c|c|c|c|c|}
\hline Nodes & $d\left(v_{a}, A_{1}\right)$ & $d\left(v_{a}, A_{2}\right)$ & $d\left(v_{a}, A_{3}\right)$ & $d\left(v_{a}, A_{4}\right)$ \\
\hline$v_{a}(a=1)$ & 0 & 2 & 1 & 2 \\
\hline$v_{a}(a=t / 2)$ & 0 & $t / 2$ & $t / 2$ & $t / 2-1$ \\
\hline$v_{a}(a=t / 2+1)$ & 0 & $t / 2-1$ & $t / 2$ & $t / 2$ \\
\hline$v_{a}(t / 2+2 \leq a \leq t-1)$ & 0 & $t-a$ & $t-a+1$ & $t-a+2$ \\
\hline$v_{a}(a=t)$ & 1 & 0 & 1 & 2 \\
\hline$v_{a}(a=t+1)$ & 1 & 1 & 0 & 1 \\
\hline$v_{a}(a=t+2)$ & 1 & 2 & 1 & 0 \\
\hline
\end{tabular}

TABLe 12: $r\left(v_{a} \mid \Psi\right)$ for $T_{n}\langle 1, t\rangle$ when $n=t+3$ with even $t \geq 4$.

\begin{tabular}{|c|c|c|c|c|}
\hline Nodes & $d\left(v_{a}, A_{1}\right)$ & $d\left(v_{a}, A_{2}\right)$ & $d\left(v_{a}, A_{3}\right)$ & $d\left(v_{a}, A_{4}\right)$ \\
\hline$v_{a}(a=1)$ & 0 & $\tau$ & $t / 2-2$ & 1 \\
\hline$v_{a}(a=2)$ & 0 & $\tau-1$ & $t / 2-1$ & 1 \\
\hline$v_{a}(3 \leq a \leq \tau)$ & 0 & $\tau-a+1$ & $t / 2-a+3$ & $a-2$ \\
\hline$v_{a}(a=\tau+1)$ & 1 & 0 & $a-2$ & $a-2$ \\
\hline$v_{a}(\tau+2 \leq a \leq t / 2+2)$ & $a-\tau$ & 0 & $t / 2-a+3$ & $t / 2-a+5$ \\
\hline$v_{a}(a=t / 2+3)$ & $\tau$ & 1 & 0 & 2 \\
\hline$v_{a}(a=n / 2+4)$ & $\tau+1$ & 2 & 0 & 1 \\
\hline$v_{a}(t / 2+5 \leq a \leq t / 2+8)$ & $a-\tau$ & $a-t / 2-2$ & $a-t / 2-4$ & 0 \\
\hline$v_{a}(t / 2+9 \leq a \leq n-7)$ & $t-a+2$ & $a-t / 2-2$ & $a-t / 2-4$ & 0 \\
\hline$v_{a}(n-6 \leq a \leq n-2)$ & $t-a+2$ & $t-a+\tau+2$ & $a-t / 2-4$ & 0 \\
\hline$v_{a}(a=n-1)$ & 1 & $\tau$ & $t / 2-2$ & 0 \\
\hline$v_{a}(a=n)$ & 1 & $\tau-1$ & $t / 2-1$ & 0 \\
\hline
\end{tabular}

TABLe 13: $r\left(v_{a} \mid \Psi\right)$ for $T_{n}\langle 1, t\rangle$ when $n=t+3$ with even $t \geq 4$.

\begin{tabular}{|c|c|c|c|c|}
\hline Nodes & $d\left(v_{a}, A_{1}\right)$ & $d\left(v_{a}, A_{2}\right)$ & $d\left(v_{a}, A_{3}\right)$ & $d\left(v_{a}, A_{4}\right)$ \\
\hline$v_{a}(a=1)$ & 0 & $\tau$ & $\tau-2$ & 1 \\
\hline$v_{a}(a=2)$ & 0 & $\tau-1$ & $\tau-1$ & 1 \\
\hline$v_{a}(3 \leq a \leq \tau-5)$ & 0 & $\tau-a+1$ & $a+\tau-3$ & $a-2$ \\
\hline$v_{a}(\tau-4 \leq a \leq \tau)$ & 0 & $\tau-a+1$ & $t / 2-a+3$ & $a-2$ \\
\hline$v_{a}(\tau+1 \leq a \leq t / 2-4)$ & $a-\tau$ & 0 & $t / 2-a+3$ & $a-2$ \\
\hline$v_{a}(t / 2-3 \leq a \leq t / 2+2)$ & $a-\tau$ & 0 & $t / 2-a+3$ & $a-\tau+1$ \\
\hline$v_{a}(t / 2+3 \leq a \leq t / 2+8)$ & $a-\tau$ & $a-t / 2-2$ & 0 & $a-\tau-1$ \\
\hline$v_{a}(t / 2+9 \leq a \leq 3 \tau)$ & $t-a+2$ & $a-t / 2-2$ & 0 & $3 \tau-a+1$ \\
\hline$v_{a}(3 \tau+1 \leq a \leq t-4)$ & $t-a+2$ & $a-t / 2-2$ & $a-3 \tau$ & 0 \\
\hline$v_{a}(t-3 \leq a \leq n-2)$ & $t-a+2$ & $3 \tau+t / 2-a$ & $a-3 \tau$ & 0 \\
\hline$v_{a}(a=n-1)$ & 1 & $\tau$ & $\tau-2$ & 0 \\
\hline$v_{a}(a=n)$ & 1 & $\tau-1$ & $\tau-1$ & 0 \\
\hline
\end{tabular}


TABLE 14: $r\left(v_{a} \mid \Psi\right)$ for $T_{n}\langle 1, t\rangle$ when $n=t+4$ with even $t \geq 4$.

\begin{tabular}{lcccc}
\hline Nodes & $d\left(v_{a}, A_{1}\right)$ & $d\left(v_{a}, A_{2}\right)$ & $d\left(v_{a}, A_{3}\right)$ & $d\left(v_{a}, A_{4}\right)$ \\
\hline$v_{a}(a=1)$ & 0 & 1 & 2 & 4 \\
$v_{a}(a=2)$ & 0 & 2 & 1 & 3 \\
$v_{a}(a=3)$ & 0 & 1 & 1 & 2 \\
$v_{a}(4 \leq a \leq t / 2+2)$ & $a-3$ & 0 & $a-2$ & $a-3$ \\
$v_{a}(a=t / 2+3)$ & $t-1$ & 0 & $t-1$ & $t$ \\
$v_{a}(t / 2+4 \leq a \leq n-3)$ & $t-a+2$ & 0 & $t-a+2$ & $t-a+4$ \\
$v_{a}(a=n-2)$ & 1 & 1 & 0 & 2 \\
$v_{a}(a=n-1)$ & 1 & 2 & 0 & 1 \\
$v_{a}(a=n)$ & 2 & 1 & 1 & 0 \\
\hline
\end{tabular}

Case 1: for $n=t+2$, let $A_{1}=\left\{v_{1}, v_{2}, v_{3}\right\}$, $A_{2}=\left\{v_{4}, v_{5}, \ldots, v_{n-2}\right\}, A_{3}=\left\{v_{n-1}\right\}, \quad$ and $A_{4}=\left\{v_{n}\right\}$. When $n=t+2$, the $r\left(v_{a} \mid \Psi\right)$ are tabulated in Table 8 .

Case 2: for $n=t+3$, let $A_{1}=\left\{v_{1}, v_{2}, v_{3}, v_{4}\right\}$, $A_{2}=\left\{v_{5}, v_{6}, \ldots, v_{n-2}\right\}, A_{3}=\left\{v_{n-1}\right\}$, and $A_{4}=\left\{v_{n}\right\}$. When $n=t+3$, the $r\left(v_{a} \mid \Psi\right)$ are tabulated in Table 9 .

Case 3: for $n=t+4$, let $A_{1}=\left\{v_{1}, v_{2}, v_{3}\right\}, A_{2}=\left\{v_{4}\right.$, $\left.v_{5}, \ldots, v_{n-3}\right\}, A_{3}=\left\{v_{n-2}, v_{n-1}\right\}$, and $A_{4}=\left\{v_{n}\right\}$. When $n=t+4$, the $r\left(v_{a} \mid \Psi\right)$ are tabulated in Table 10 .

Tables $8-10$ clearly show that $\Psi$ is a 2 -resolving generator of $T_{n}\langle 1, t\rangle$ for $n=t+2, t+3, t+4$, therefore $\operatorname{pd}_{2}\left(T_{n}\langle 1, t\rangle\right) \leq 4$ for $n=t+2, t+3, t+4$. Lemma 1 implies that $\operatorname{pd}_{2}\left(T_{n}\langle 1, t\rangle\right) \geq 4$ for $n=t+2, t+3, t+4$. This establishes our claim.

Theorem 5. Consider $T_{n}\langle 1, t\rangle$ with even $t \geq 4$, then $p d_{2}\left(T_{n}\langle 1, t\rangle\right)=4$, for $n=t+2, t+3, t+4$.

Proof. Let $\Psi=\left\{A_{1}, A_{2}, A_{3}, A_{4}\right\}$ be a partition of $V\left(T_{n}\langle 1, t\rangle\right)$. The proof consists of three parts.

Case 1: for $n=t+2$, let $A_{1}=\left\{v_{1}, v_{2}, \ldots, v_{n-3}\right\}$, $A_{2}=\left\{v_{n-2}\right\}, A_{3}=\left\{v_{n-1}\right\}, \quad$ and $A_{4}=\left\{v_{n}\right\}$. When $n=t+2$, the $r\left(v_{a} \mid \Psi\right)$ are tabulated in Table 11 .

Case 2: for $n=t+3$, let $\tau=\lceil n / 4\rceil$.

Case 2a: when $n \equiv 1(\bmod 4)$, let $A_{1}=\left\{v_{a} \mid 1 \leq a \leq \tau\right\}$, $A_{2}=\left\{v_{a} \mid \tau+1 \leq a \leq 2 \tau-1\right\}, A_{3}=\left\{v_{a} \mid 2 \tau \leq a \leq 2 \tau+1\right\}$, and $A_{4}=\left\{v_{a} \mid 2 \tau+2 \leq a \leq n\right\}$.

When $n=t+3$, the $r\left(v_{a} \mid \Psi\right)$ are tabulated in Table 12 . Case $2 \mathrm{~b}$ : when $n \equiv 3(\bmod 4)$, let $A_{1}=\left\{v_{a} \mid 1 \leq a \leq \tau\right\}$, $A_{2}=\left\{v_{a} \mid \tau+1 \leq a \leq 2 \tau\right\}, \quad A_{3}=\left\{v_{a} \mid 2 \tau+1 \leq a \leq 3 \tau\right\}$, and $A_{4}=\left\{v_{a} \mid 3 \tau+1 \leq a \leq n\right\}$.

When $n=t+3$, the $r\left(v_{a} \mid \Psi\right)$ are tabulated in Table 13 .

Case 3: for $n=t+4$, let $A_{1}=\left\{v_{1}, v_{2}, v_{3}\right\}, A_{2}=\left\{v_{4}\right.$, $\left.v_{5}, \ldots, v_{n-3}\right\}, A_{3}=\left\{v_{n-2}, v_{n-1}\right\}$, and $A_{4}=\left\{v_{n}\right\}$. When $n=t+4$, the $r\left(v_{a} \mid \Psi\right)$ are tabulated in Table 14 .

It is clear from Tables $11-14$ that $\Psi$ is a 2 -resolving generator of $T_{n}\langle 1, t\rangle$ for $n=t+2, t+3, t+4$, therefore $\operatorname{pd}_{2}\left(T_{n}\langle 1, t\rangle\right) \leq 4$ for $n=t+2, t+3, t+4$. Lemma 1 implies that $\operatorname{pd}_{2}\left(T_{n}\langle 1, t\rangle\right) \geq 4$ for $n=t+2, t+3, t+4$. This establishes our claim.

\section{Conclusion}

In this research article, we conclude that the fault tolerant partition dimension of $T_{n}\langle 1, t\rangle$ is 4 when $t=2$ and $n \geq 4$. When $t=3$ and $n \in\{5,6, \ldots, 13\}$, the $\mathrm{pd}_{2}$ is 4 and its value is less than or equal to 5 for $n \geq 14$. It is also inferred that the fault partition dimension of Toeplitz networks $T_{n}\langle 1, t\rangle$ is 4 when $n=t+2, t+3, t+4$ for even $t \geq 4$ and odd $t \geq 5$ [18].

Open Problem 1. Calculate the $\operatorname{pd}_{2}\left(T_{n}\langle 1, t\rangle\right)$ for odd $t \geq 5$ and $n \geq t+5$.

Open Problem 2. Calculate the $\operatorname{pd}_{2}\left(T_{n}\langle 1,2, t\rangle\right)$ for $t \geq 3$.

\section{Data Availability}

All data are included within this article. However, the reader may contact the corresponding author for more details of the data.

\section{Conflicts of Interest}

The authors declare that they have no conflicts of interest.

\section{References}

[1] N. Deo and M. S. Krishnamoorthy, "Toeplitz networks and their properties," IEEE Transactions on Circuits and Systems, vol. 36, no. 8, pp. 1089-1092, 1989.

[2] P. J. Slater, "Leaves of trees," Proc. 6th Southeastern Conf. Combinatorics, Graph Theory and Computing, Congressus Numerantium, vol. 14, pp. 549-559, 1975.

[3] F. Harary and R. A. Melter, "On the metric dimension of a graph," Theory Comput. Syst. Ars Combinatoria.vol. 2, pp. 191-195, 1976.

[4] S. Khuller, B. Raghavachari, and A. Rosenfeld, "Landmarks in graphs," Discrete Applied Mathematics, vol. 70, no. 3, pp. 217-229, 1996.

[5] G. Chartrand, L. Eroh, M. A. Johnson, and O. R. Oellermann, "Resolvability in graphs and the metric dimension of a graph," Discrete Applied Mathematics, vol. 105, no. 1-3, pp. 99-113, 2000.

[6] C. Hernando, M. Mora, P. J. Slater, and D. R. Wood, "Faulttolerant metric dimension of graphs," in proceedings international conference on convexity in discrete structures; Ramanujan mathematical society, pp. 81-85, Tiruchirappalli, India, 2008.

[7] A. Estrado-Moreno, J. A. Rodriguez-Velaquez, and I. G. Yero, "The $k$ - metric dimension of a graph," Appl. Math. Inf. Sci.vol. 9, no. 6, pp. 2829-2840, 2015.

[8] J. B. Liu, M. Munir, I. Ali, Z. Hussain, and A. Ahmed, FaultTolerant Metric Dimension of Wheel Related Graphs, 2019.

[9] I. G. Yero, A. Estrada-Moreno, and J. A. RodríguezVelázquez, "Computing the $\mathrm{k}$-metric dimension of graphs $k-$ metric dimension of graphs," Applied Mathematics and Computation, vol. 300, pp. 60-69, 2017.

[10] G. Chartrand, E. Salehi, E. Salehi, and P. Zhang, "The partition dimension of a graph," Aequationes Mathematicae, vol. 59, no. 1 , pp. $45-54,2000$. 
[11] I. Javaid, M. Salman, M. A. Chaudhry, and S. Shokat, "Fault tolerance in resolvability," Utilitas Mathematica, vol. 80, pp. 263-275, 2009.

[12] H. Fernau, J. A. Rodriguez-Velaquez, and I. G. Yero, "On the partition dimension of unicyclic graphs," Bulletin Mathématique De La Société Des Sciences Mathématiques De Roumanie, Nouvelle Série, vol. 57, no. 105, pp. 381-91, 2014, https://arxiv.org/abs/1111.3513.

[13] K. Q. Fredlina and E. T. Baskoro, "The partition dimension of some families of trees," Procedia Computer Science, vol. 74, pp. 60-66, 2015.

[14] A. Estrado-Moreno, "On the $k$ - partition dimension of graphs," Theoretical Computer Science, vol. 806, pp. 42-52, 2020.

[15] K. Azhar, S. Zafar, A. Kashif, and Z. Zahid, "On fault-tolerant partition dimension of graphs," Journal of Intelligent \& Fuzzy Systems, vol. 40, no. 1, pp. 1129-1135, 2021.

[16] A. Nadeem, A. Kashif, S. Zafar, and Z. Zahid, "On 2-partition dimension of the circulant graphs," Journal of Intelligent \& Fuzzy Systems, vol. 40, no. 5, pp. 9493-9503, 2021.

[17] J.-B. Liu, M. F. Nadeem, H. M. A. Siddiqui, and W. Nazir, "Computing metric dimension of certain families of Toeplitz graphs," IEEE Access, vol. 7, Article ID 126734, 2019.

[18] M. R. Garey and D. S. Johnson, Computers and Intractability: A Guide to the Theory of NP-Completeness, Freeman, New York, 1979. 